\title{
Fatty acid composition of Drosophila photoreceptor light-sensitive microvilli
}

\author{
Yorka Muñoz ${ }^{\mathrm{a}}$, Karen Fuenzalidab ${ }^{\mathrm{b}}$ Miguel Bronfmann ${ }^{\mathrm{b}}$, Arnaldo Gaticac, Marcelo Sepúlveda ${ }^{\mathrm{a}}$ Juan Bacigalupo ${ }^{\mathrm{a}}$ \\ Alejandro D. Roth ${ }^{\mathrm{a}}$ and Ricardo Delgado ${ }^{\mathrm{a}^{*}}$ \\ a Department of Biology, Faculty of Sciences, University of Chile, Las Palmeras 3425, Santiago 7800024, Chile. \\ ${ }^{b}$ Faculty of Biological Sciences, Pontifical Catholic University of Chile, Libertador Bernardo O'Higgins 340 Santiago 8331150, Chile. \\ ${ }^{c}$ Institute of Nutrition and Food Technology, University of Chile, El Líbano 5524, Santiago 7830490, Chile.
}

\begin{abstract}
Phototransduction, the mechanism underlying the electrical response to light in photoreceptor cells, has been thoroughly investigated in Drosophila melanogaster, an essential model in signal transduction research. These cells present a highly specialized photosensitive membrane consisting of thousands of microvilli forming a prominent structure termed a rhabdomere. These microvilli encompass the phototransduction proteins, most of which are transmembrane and exclusively rhabdomeric. Rhabdomere membrane lipids play a crucial role in the activation of the transient receptor potential ionic channels (TRP and TRPL) responsible for initiating the photoresponse. Despite its importance, rhabdomere lipid composition has not been established. We developed a novel preparation enriched in rhabdomere membranes to perform a thorough characterization of the lipidomics of Drosophila rhabdomeres. Isolated eyes (500) were homogenized and subjected to a differential centrifugation protocol that generates a fraction enriched in rhabdomere membrane. Lipids extracted from this preparation were identified and quantified by gas chromatography coupled to mass spectrometry. We found an abundance of low sterol esters (C16:0, C18:0), highly abundant and diverse triglycerides, free fatty acids, a moderate variety of mono and diacyglycerols (C:16:0, 18:0, C18:1) and abundant phospholipids (principally C18:2). This preparation opens a new avenue for investigating essential aspects of phototransduction.
\end{abstract}

Key terms: Rhabdomere, Drosophila, microvilli, phototransduction, TRP channel, fatty acid analysis.

\section{INTRODUCTION}

The concept of lipid functions has long surpassed the roles of static energy reserves or passive membrane components, as their participation in multiple signaling pathways and central roles in the organization and modulation of membrane protein functions is widely recognized. Aided by their structural diversity in chain length, saturation level and polar head, lipids participate in the establishment of membrane domains such as lipid rafts, segregating proteins according to the thermodynamic interactions of intra-membrane protein segments with their surrounding lipids (Brown and London, 1998; Munro, 1995). The local lipid environment in which membrane proteins are embedded can often be determinant in their specific function (Boesze-Battaglia and Schimmel, 1997).

A remarkable case of the participation of membrane lipids in signaling cascades is that of light transduction in Drosophila melanogaster photoreceptors, one of the fastest signal transduction mechanisms known. This process takes place in a specialized, prominent and segregated membrane structure of each photoreceptor cell termed rhabdomeres, which consists of a tight arrangement of $\sim 50,000$ microvilli (Wang and Montell, 2007). These organelles are free of internal membranes and possess an inner arrangement of $\beta$-actin filaments. The main proteins that participate in phototransduction, most of which are transmembrane, are confined to the rhabdomere. It should be remarked that the characterization of Drosophila phototransduction cascade led, among other findings, to the discovery and cloning of the founding member of the vast superfamily of the TRP cation channels (Montell and Rubin, 1989; Hardie and Minke, 1992). Many TRP channels have key roles in signal transduction in a variety of primary sensory receptor cells (Clapham, 2003). In fly phototransduction, photoisomerized rhodopsin couples to a GTP-binding protein, which activates phospholipase- $\mathrm{C}_{\beta}$ (PLC). This key enzyme (Estacion et al. 2001) catalyzes the hydrolysis of phosphatidylinositol 4,5-bisphosphate ( $\left.\mathrm{PIP}_{2}\right)$ into 1,4,5-inositol trisphosphate $\left(\mathrm{InsP}_{3}\right)$ and diacylglycerol (DAG) (Hardie and Postma, 2008). The mechanism that links these events to the activation of transient receptor potential (TRP) channels remains to be determined, particularly because the participation of $\mathrm{InsP}_{3}$ has been ruled out (Lev et al. 2012; Raghu et al. 2012). Therefore the possible roles of $\mathrm{PIP}_{2}$, DAG or polyunsaturated fatty acids, presumably derived from DAG hydrolysis by a putative DAG-lipase (Leung et al., 2008), in the opening the TRP channels is currently a matter of intensive debate. However, in spite of the fundamental relevance that the lipid composition of the photosensitive membrane has for Drosophila phototransduction, the lipid environment where this cascade is carried out has not been determined.

Lipid metabolism in Drosophila is markedly different from that of vertebrates (see Hammad et al. 2011, Tortoriello et al., 2013). The establishment of the rhabdomere lipid composition is key for advancing in the characterization of the putative lipid metabolites that participate in TRP activation in response to light. It is remarkable that a Drosophila rhabdomere membrane preparation suitable for biochemical studies such as lipidomics is not available. To date lipidomic characterizations 
of Drosophila fatty acids have been performed on entire flies or heads (Carvalho et al. 2012; Hammad et al. 2011; Shen et al. 2010), while studies on Drosophila retinas have focused on the levels of general lipid components and have not determined the presence or abundance of specific fatty acids (Fujita et al. 1987; Garcia-Murillas et al. 2006; Raghu et al. 2009).

Starting from isolated eyes, we used differential centrifugation to fractionate Drosophila retinas in order to obtain a sample enriched in rhabdomere membranes, as evaluated by the presence of specific protein markers. Folch total lipid extracts from this sample were fractionated into major lipid classes according to their differential retention in aminopropyl columns (Agren et al. 1992). Fatty acid profiles of each lipid class were analyzed using gas chromatographymass spectrometry (GC-MS). This novel membrane preparation allowed us to achieve the first characterization of the fatty acid composition of the rhabdomere membrane lipids of Drosophila. This preparation will contribute significantly to the study of Drosophila phototransduction.

\section{METHODS}

Preparation of a membrane fraction enriched in rhabdomeres

All membrane preparations derived from a white-eyed Oregon- $\mathrm{R}$ strain. Colony founding flies were kindly provided by Dr. Craig Montell (Johns Hopkins University, USA). Animals were fed with standard Drosophila food composed of yeast $(10 \mathrm{~g} / \mathrm{L})$, corn $(60$ $\mathrm{g} / \mathrm{L})$, agar $(10 \mathrm{~g} / \mathrm{L})$, sucrose $(43 \mathrm{~g} / \mathrm{L}), \mathrm{NaNO}_{3}(4.2 \mathrm{~g} / \mathrm{L}), \mathrm{K}_{2} \mathrm{HPO}_{4}$ (1.2 g/L), $\mathrm{KCl}(0.57 \mathrm{~g} / \mathrm{L}), \mathrm{MgSO}_{4}(0.57 \mathrm{~g} / \mathrm{L}), \mathrm{FeSO}_{4}(0.013 \mathrm{mg} / \mathrm{L})$ (modified from Lewis, 1960) and kept at $18{ }^{\circ} \mathrm{C}$ in glass vials under 12 hour light-dark cycles. Animal management, bio-safety and experimental procedures were in accordance with the bioethics and animal welfare committee of the Faculty of Science of the University of Chile. For each preparation 500 eyes were dissected from adult flies previously anesthetized with $\mathrm{CO}_{2}$, collected into pre-chilled $1.5 \mathrm{~mL}$ tubes containing cold divalent cation-free Drosophila Ringer and stored at $-80{ }^{\circ} \mathrm{C}$ until processed. Frozen eyes were mechanically disrupted in a Mini-bead beater (model 3110BXEUR, Biospec products, Bartlesville, OK, USA; $0.1 \mathrm{~mm}$ glass beads) by agitation at maximum speed 10 sequential times of $1 \mathrm{~min}$ periods separated by $40 \mathrm{~s}$ cooling of the homogenization vial in ice, to prevent damage to the sample. This operation completely disrupted the tissues, as confirmed by microscopic evaluation of sample smears (40x, Nikon TMS inverted microscope. Nikon, Japan).

\section{Differential Centrifugation}

The homogenate $(\mathrm{H})$ was spun in a tabletop centrifuge at $10 \mathrm{xg}$ for $10 \mathrm{~min}$ at $4{ }^{\circ} \mathrm{C}$ to precipitate the debris. The supernatant S1 was centrifuged at 2,370xg for $5 \mathrm{~min}$ at $4{ }^{\circ} \mathrm{C}$; the resulting pellet P2 was saved and the supernatant S2 was centrifuged at $18,620 \mathrm{xg}$ for $15 \mathrm{~min}$ at $4{ }^{\circ} \mathrm{C}$, yielding the supernatant $\mathrm{S} 3$ and the pellet P3. Each sample was snap-frozen in liquid nitrogen and stored at $-80{ }^{\circ} \mathrm{C}$ until use.

\section{Immunoblot analysis}

Samples were resuspended in cation-free Drosophila Ringer containing anti-proteases (complete-EDTA free antiprotease cocktail, Roche, NY, USA) and protein concentration was determined colorimetrically (Hartree, 1972). Samples of
$20 \mu \mathrm{g}$ total protein were subjected to PAGE in $7 \%$ and $10 \%$; polyacrylamide gels were run at $100 \mathrm{~V}$ for 1.5 hours. Proteins were transferred to nitrocellulose membranes at $4{ }^{\circ} \mathrm{C}$ in 40 $\mathrm{mM}$ TRIS/glycine $(\mathrm{pH} 8.8)+10 \%$ methanol and $20 \%$ methanol $(\mathrm{v} / \mathrm{v})$, respectively, for 1.5 hour at $300 \mathrm{~mA}$. Nitrocellulose membranes were blocked with $5 \%$ non-fat dry milk in TRIS-buffered saline supplemented with $0.05 \%$ Tween-20 (TBS-T) for 2 hours at room temperature and incubated with primary antibodies overnight at $4{ }^{\circ} \mathrm{C}$. Antibodies were the following: anti-TRP 1:200 (rhabdomere membrane marker; Developmental Studies Hybridoma Bank, University of Iowa, Iowa City, IA, USA), anti-lamin Dmo 1:200 (nucleus marker; Developmental Studies Hybridoma Bank, University of Iowa,); anti- $\beta$-actin 1:1,000 (rhabdomere membrane marker, Sigma-Aldrich, St. Louis, MO, USA) and anti-SOD2 1:200 (mitochondrial marker; Abcam, Cambridge, UK). Membranes were washed five times in TBS-T for $5 \mathrm{~min}$ and then incubated for 2 hours at room temperature with secondary antibodies conjugated with horseradish peroxidase: goat anti-mouse IgGHRP 1:10,000 and goat anti-rabbit IgG-HRP 1:5,000 (Thermo Scientific, Waltham, MA, USA), in blocking solution. Finally, blots were washed 5 times in TBS-T for $5 \mathrm{~min}$ and signals were detected using the enhanced ECL kit (Pierce, Thermo Scientific, Waltham, MA, USA) according to manufacturer's instructions.

Rhabdomere lipid extraction, separation and fatty acid methyl esters (FAME) analysis

Internal standards FFA-C19:0 or, phospho-C17 and TAG-C15 (5 $\mu$ g each) were added to P2 fractions prior to Folch total lipid extraction using 2:1 chloroform to methanol (Folch et al. 1957). Different lipid species were separated by aminopropyl columns (Agren et al. 1992) and fatty acid methyl esters were obtained by overnight incubation at $50{ }^{\circ} \mathrm{C}$ with $1 \mathrm{~N} \mathrm{HCl}$ in anhydrous methanol. $2 \mathrm{~mL}$ sodium bicarbonate solution $\left(6 \% \mathrm{w} / \mathrm{v}\right.$ in $\left.\mathrm{H}_{2} \mathrm{O}\right)$ were added and FAME were extracted with $2 \mathrm{~mL}$ hexane. The remaining water phase was washed with $2 \mathrm{~mL}$ hexane, and both hexane extracts were dried under a nitrogen stream. Fatty acid methyl esters were re-dissolved in hexane $(500 \mu \mathrm{L})$ and $5 \mu \mathrm{L}$ samples were injected into a gas chromatograph (Hewlett Packard HP 5890 series II; Palo Alto, CA, USA) coupled to a mass spectrometer (Hewlett Packard 5972). The gas chromatograph was equipped with an HP-INNOWax, 25 $\mathrm{m} \times 0.2 \mathrm{~mm} \times 0.2 \mu \mathrm{m}$ capillary column (Agilent Tech, Santa Clara, CA, USA). The oven temperature was programmed for a temperature ramp of $1 \mathrm{~min}$ at $150^{\circ} \mathrm{C}$, then $15^{\circ} \mathrm{C} / \mathrm{min}$ to $200{ }^{\circ} \mathrm{C}$ and $12{ }^{\circ} \mathrm{C} / \mathrm{min}$ to $260{ }^{\circ} \mathrm{C}$. The injector and mass spectrometer detector temperatures were $220^{\circ} \mathrm{C}$ and $275{ }^{\circ} \mathrm{C}$, respectively. Helium was used as carrier gas at a flow rate of $1 \mathrm{~mL} /$ min; sample injection was done in splitless mode. Fatty acid composition is expressed as a percentage of total fatty acids.

Relative fatty acid (FA) composition was quantified by comparison to internal standard peaks (FFA-19:0 and FFA-17:0) and normalized to sample mass.

\section{RESULTS}

Drosophila ommatidia fractionation yields a fraction enriched in rhabdomere membrane

In order to obtain a suspension of Drosophila rhabdomere membranes, we adopted a homogenization protocol using a Mini-bead beater, which unlike Dounce tissue-grinders or 
N2-snap freeze and crushing with a porcelain mortar, yielded adequate tissue disruption and sample recovery. Disrupted tissue was allowed to settle before retrieving the homogenate preparation $(\mathrm{H})$, which was fractionated under different centrifugation protocols. The distribution of specific marker proteins helped to determine rhabdomere presence and enrichment in each fraction. Figure 1 (track $\mathrm{H}$ ) corresponds to tissue debris and nuclear membranes, the latter revealed by the enrichment of the nuclear protein lamin in the precipitate after a slow centrifugation step (10xg, $10 \mathrm{~min})$. The resulting pellet (P1) presents a significant amount of rhabdomere membranes, as ascertained by the presence of TRP. The next centrifugation step $(2,370 \mathrm{xg}, 5 \mathrm{~min})$ generated a pellet (P2) that was further enriched in TRP and separated from the majority of SOD-2 rich mitochondrial-marker, which precipitated after an additional centrifugation (not shown). P2 was also the fraction most enriched in $\beta$-Actin, consistent with the characteristic abundance of actin filaments in microvilli (Arikawa et al. 1990).

Although there is some contamination of P2 by nuclear membranes, as shown by the presence of lamin Dmo (compare lanes P1 versus P2 in figure 1B), it should be noted that the signal for lamin Dmo is significantly decreased in P2 compared to $\mathrm{H}$ or P1. Therefore, although nuclear membrane lipids are present, they are far less represented in the rhabdomere membraneenriched sample. The difference in membrane (and therefore lipid) abundance between rhabdomeres and nuclei is staggering. Considering a nucleus radius of $0.5 \mu \mathrm{m},(\mathrm{x} 2$ taking into account its inner and outer membranes) and that each ommatidium is made up of 20 cells ( 8 photoreceptors and 12 pigment cells), the nuclear membrane surface for the retina composed of 800 ommatidia would be $\approx 6 \times 10^{4} \mu \mathrm{m}^{2}$, compared to a total rhabdomere membrane $(50,000$ microvilli per rhabdomere, each $1.5 \mu \mathrm{m}$ long and $0.05 \mu \mathrm{m}$ in diameter), of $\approx 3.3 \times 10^{6} \mu \mathrm{m}^{2}$ per eye, we estimate that nucleus membranes represent at most $1 \%$ of

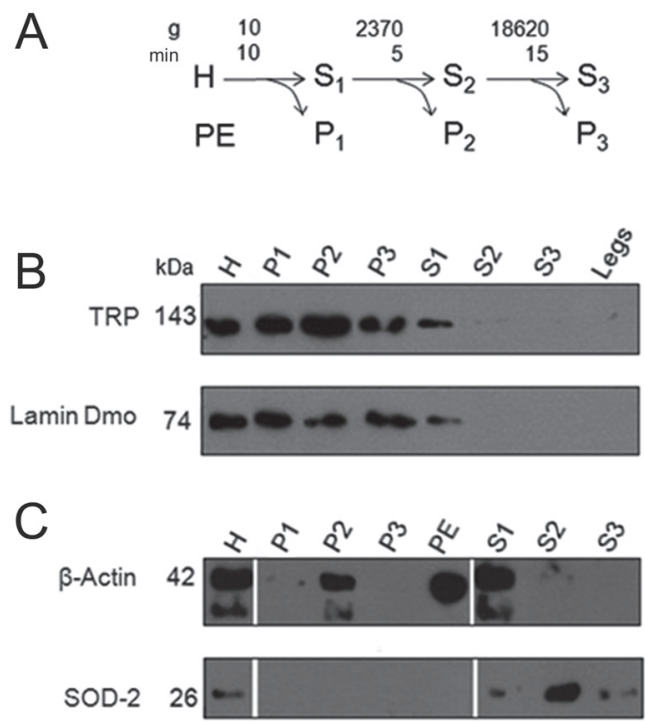

Figure 1: Procedure for obtaining a Drosophila membrane fraction enriched in rhabdomeres. A: Fractionation protocol. B: Immunoblot of the different fractions showing immunoreaction for the following proteins: TRP, rhabdomere marker; lamin, nucleus marker; $\beta$-Actin, microvilli (rhabdomere) marker; superoxide dismutase 2 , mitochondrial marker. H: crude homogenate: PE: glass-beads, cell debris and chitin sedimentation pellet. P1- P3: pellets. S1-S3: soluble fractions. the rhabdomere membranes (size considerations as reported in Wang and Montell, 2007). Our estimate assumes that other parts of the visual system were not attached to the eyes, as suggested by visual inspection of the extracted organs. If some of this material was present, it was minimally represented.

\section{Characterization of Drosophila rhabdomere membrane lipids}

The lipid species from the P2 fraction were separated by differential elution from aminopropyl columns, trans-esterified to obtain FAME, separated and identified by GC-MS (see Methods). Representative chromatographs of each fraction are shown in Figure 2, where chromatogram order (A-E) reflects increasing polarity of the solvents used to elute from aminopropyl columns.
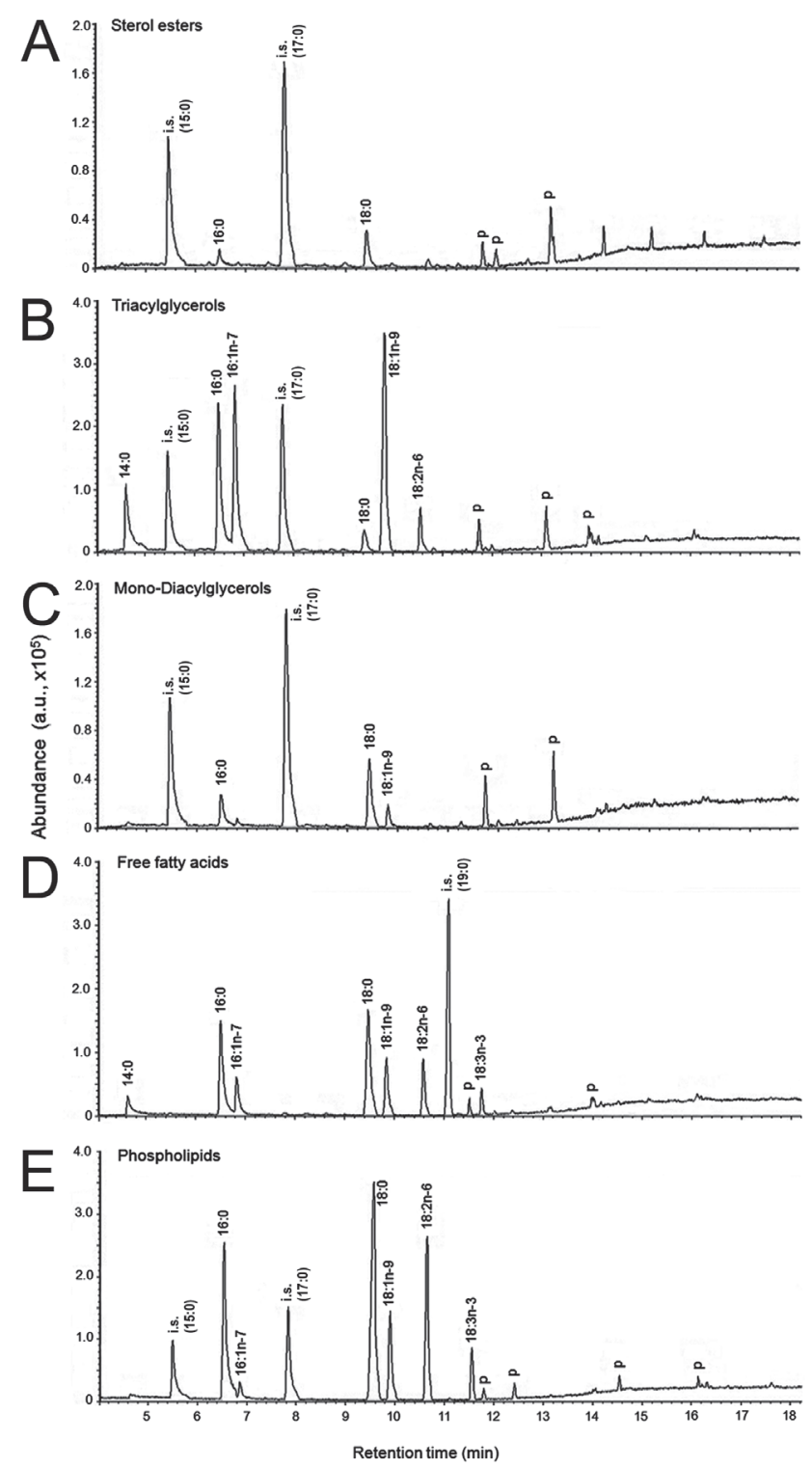

Figure 2: Representative fatty acid composition chromatograms of lipids from rhabdomere fraction (P2). A, sterol esters (SE). B, triacylglycerols. $C$, mono and diacylglycerols. D, free fatty acids. E, phospholipids. Peaks at 15:0 and 17:0 correspond to internal standards for SE, TAG, MDAG and PL fractions. 19:0 is the internal standard for FFA fraction, $\mathrm{p}$ denotes plastic contaminants. 
Sterol esters fractions contained only C16:0 and C18:0 fatty acids, which were consistently found in all samples $(n=5)$, albeit in low abundance (Figure 2 and Tables 1 \& 2) compared to other lipid fractions. Triglycerides presented diverse acyl-chain lengths and high abundance, particularly monounsaturated fatty acids C16:1 and C18:1, but with a significant contribution of C16:0 saturated fatty acids (Table 1).

Fatty acid composition of mono and diacylglycerol lipids (MDAGs) was represented by only three fatty acid species: C16:0, C18:0 and C18.1 (Tables 1 and 2), while freefatty acids (FFAs) were the most diverse lipid class in this sample, presenting multiple saturated (C14:0; C16:0; C18:0), monounsaturated $(\mathrm{C} 16: 1 ; \mathrm{C} 18: 1)$ and polyunsaturated (C18:2 and C18:3) fatty acids. All FFA were detected at intermediate or abundant levels compared to other fractions. Phospholipids in the rhabdomere contained significant amounts of C16:0 and C18:0 saturated fatty acids and C18 derived monounsaturated and polyunsaturated fatty acids C18:1 and C18:2. Remarkably, C18:3 was present almost exclusively in the PL fraction.

\section{DISCUSSION}

Over the last few years there has been an explosive progress in subcellular fractionation methods in parallel with an increase in sensitivity of high-throughput analysis techniques. The interdependence of these fields is well documented in proteomic characterizations of cellular and subcellular fractions. These technological improvements have allowed a dramatic refinement in the resolution of the measurements of molecular components in spatially confined cellular regions, previously obscured by contamination from adjacent cellular compartments with which they were inevitably associated in the biological samples. Thus although there are good characterizations of the general lipid

TABLE 1

Fatty acid composition of different lipid classes in Drosophila rhabdomere membranes ${ }^{a}$

\begin{tabular}{|c|c|c|c|c|c|c|c|}
\hline \multirow[b]{2}{*}{ Rhabdomere } & \multicolumn{7}{|c|}{ Fatty acid $\%$} \\
\hline & 14:0 & $16: 0$ & $16: 1$ & $18: 0$ & 18:1 & $18: 2$ & $18: 3^{a}$ \\
\hline $\mathrm{SE}^{\mathrm{b}}$ & ND & $\begin{array}{l}37.6 \\
\pm 5.1\end{array}$ & ND & $\begin{array}{l}62.4 \\
\pm 5.2\end{array}$ & ND & ND & ND \\
\hline $\mathrm{TAG}^{\mathrm{c}}$ & $\begin{array}{l}17.5 \\
\pm 1.6\end{array}$ & $\begin{array}{l}27.7 \\
\pm 2.1\end{array}$ & $\begin{array}{l}20.9 \\
\pm 3.2\end{array}$ & $\begin{array}{l}1.3 \\
\pm 0.3\end{array}$ & $\begin{array}{l}25.4 \\
\pm 1.0\end{array}$ & $\begin{array}{l}7.2 \\
\pm 1.9\end{array}$ & ND \\
\hline M-DAG ${ }^{\mathrm{d}}$ & ND & $\begin{array}{l}33.1 \\
\pm 2.4\end{array}$ & ND & $\begin{array}{l}41.6 \\
\pm 6.7\end{array}$ & $\begin{array}{l}25.3 \\
\pm 6.6\end{array}$ & ND & ND \\
\hline $\mathrm{FFA}^{\mathrm{e}}$ & $\begin{array}{l}7.2 \\
\pm 1.3\end{array}$ & $\begin{array}{l}23.3 \\
\pm 3.6\end{array}$ & $\begin{array}{l}9.4 \\
\pm 2.4\end{array}$ & $\begin{array}{l}22.9 \\
\pm 4.9\end{array}$ & $\begin{array}{l}19.4 \\
\pm 2.8\end{array}$ & $\begin{array}{l}15.7 \\
\pm 5.8\end{array}$ & $\begin{array}{l}2.0 \\
\pm 0.9\end{array}$ \\
\hline$P L^{f}$ & ND & $\begin{array}{l}18.5 \\
\pm 1.2\end{array}$ & $\begin{array}{l}2.9 \\
\pm 0.9\end{array}$ & $\begin{array}{l}13.5 \\
\pm 2.1\end{array}$ & $\begin{array}{l}22.0 \\
\pm 1.6\end{array}$ & $\begin{array}{l}36.9 \\
\pm 2.4\end{array}$ & $\begin{array}{l}6.2 \\
\pm 1.9\end{array}$ \\
\hline
\end{tabular}

aValues reflect an average of five analyses with \pm .s.e.m. N.D $=$ not detected.

bSE, sterol esters;

TAG, triacylglycerols;

dM-DAGs, mono and diacylglycerols;

eFFA, free fatty acid;

${ }^{f} \mathrm{PL}$, phospholipids. and fatty acid composition of Drosophila tissues (Carvalho et al. 2012; Hammad et al. 2011; Shen et al. 2010), there is an evident lack of information regarding the fatty acids that make up the specific lipid species present in Drosophila photoreceptor rhabdomere membranes; this represents a serious handicap for the understanding of the mechanism underlying light transduction (recently reviewed in Raghu et al. 2012). Although the phospholipid composition of these membranes has been characterized and the balance between phosphatidic acid and phosphatidylinositol species linked to both rhabdomere biogenesis (Raghu et al., 2006) and degeneration (García-Murillas et al., 2006), there are no data on the fatty acid composition of these tissues, which is crucial in the understanding of the mechanisms of activation of TRP channels and phototransduction accessory proteins embedded in this lipid-rich environment (see Raghu et al. (2012) and references therein). It is remarkable that almost no biochemistry has been performed in the study of Drosophila phototransduction, mainly due to the lack of a suitable rhabdomere preparation. The bulk of the current information about this complex molecular mechanism derives from electrophysiological, genetic and immunochemical experiments. After achieving the characterization of the rhabdomere lipidomic as the first product of the rhabdomere preparation described here, interest will obviously turn to establishing which of the lipids that have been linked to the opening of TRP ( PIP $_{2}$, DAG, PUFA) undergo light-induced changes in the rhabdomere. Such information, which is beyond the scope of the present work, would be an essential supplement to the available physiological data towards a full understanding of Drosophila phototransduction; it can now be addressed with the rhabdomere membrane preparation that we have developed.

Vertebrate photoreceptor-disk membranes (rod outer segments) originate as plasma membrane evaginations, which become deficient in cholesterol and saturated fatty acids as they mature (for review see: Albert and Boesze-Battaglia, (2005)); they are particularly enriched in VLCH-FAs and PUFAs, principally docosahexaenoic acid (DHA). These lipid species are considered to influence phototransduction events by allowing alteration of membrane topology upon rhodopsin photo-isomerization, while serving as quenchers of free radical-species formed by photon exposure (BoeszeBattaglia and Schimmel, 1997; Litman and Mitchell, 1996; McMahon and Kedzierski, 2010; Sanchez-Martin et al. 2013). In contrast, Drosophila tissues lack medium and long chain PUFAs (Hammad et al. 2011; Shen et al. 2010), and as the current study shows, its rhabdomeres do not contain fatty acids over 18 carbons and are slightly enriched in saturated FA (Table 2 ); these features would reduce membrane fluidity through higher PL compaction. Still, PL composition of rhabdomere membranes shows the highest levels of polyunsaturated fatty acids (C18:2 and C18:3), suggesting that in the absence of very long chain highly unsaturated fatty acids such as DHA, Drosophila uses C18 unsaturated fatty acids to overcome partially membrane compaction.

Another salient feature of the Drosophila rhabdomere is its TAG-derived fatty acid content and composition (Table 2), dramatically different than vertebrates. Rhabdomere TAG contains significant amounts of short chain saturated and unsaturated fatty acids (C14:0, C16:0 and C16:1), very low amounts of $\mathrm{C} 18: 0$ and $\mathrm{C} 18: 2$ and abundant C18:1. Vertebrate 
photoreceptor membranes do not contain TAG, which are abundant only in lipogenic cells such as adipocytes. Vertebrate TAG contains almost equivalent amounts of C16:0, C18:0 and C18:1. Drosophila rhabdomere TAG composition has a greater resemblance to plant seed TAGs, which also present low levels of saturated fatty acids and high levels of polyunsaturated fatty acids with different chain lengths and unsaturations (Li-Beisson et al. 2013). The biological significance of TAG in rhabdomeres is a challenging question that exceeds the scope of this work. However, considering the short chains and degree of unsaturation of the fatty acids of rhabdomere TAGs, these molecules should behave as liquid vegetable oils rather than as solid vertebrate fats, thus contributing to membrane fluidity rather than increasing its rigidity.

In terms of lipid signaling associated with phototransduction, although TRP-mediated currents can be elicited by arachidonic (AA, C20:4) and other fatty acids in photoreceptors under whole cell recording conditions in heterologous expression systems (Chyb et al. 1999) and in excised rhabdomere membrane patches (Delgado and Bacigalupo, 2009), AA was undetectable in Drosophila heads and tissues (Hammad et al. 2011; Yoshioka et al. 1985). Therefore it is unlikely that this lipid is an endogenous TRP activator, while the FA composition of PL (phospholipid) and TAG in the rhabdomere indicates that a putative TRP fatty acid activator should be an 18 carbon FA and highlights the importance of establishing light-dependent rhabdomere lipid dynamics in further studies. In vertebrate photoreceptors a cholesterol-rich environment in the disks reduces the efficiency of the phototransduction cascade by hindering the activation of rhodopsin and affecting the cyclic nucleotide phosphodiesterase activity that hydrolyzes cyclic GMP (for a comprehensive review on the role of cholesterol in rod outer segment membranes, see Albert and Boesze-Battaglia, (2005).

TABLE 2

Quantification of fatty acid of five different lipid classes of Drosophila rhabdomere membranes ${ }^{\mathrm{a}}$

\begin{tabular}{|c|c|c|c|c|c|c|c|}
\hline \multirow[b]{2}{*}{ Rhabdomere } & \multicolumn{7}{|c|}{ Fatty acid nmol/mg sample } \\
\hline & 14:0 & 16:0 & 16:1 & 18:0 & 18:1 & 18:2 & $18: 3^{a}$ \\
\hline$S E^{b}$ & ND & $\begin{array}{l}12.3 \\
\pm 4.3\end{array}$ & ND & $\begin{array}{l}20,4 \\
\pm 4.0\end{array}$ & ND & ND & ND \\
\hline $\mathrm{TAG}^{\mathrm{c}}$ & $\begin{array}{l}379.2 \\
\pm 50.3\end{array}$ & $\begin{array}{l}602.3 \\
\pm 80.1\end{array}$ & $\begin{array}{l}452.5 \\
\pm 85.0\end{array}$ & $\begin{array}{l}28.6 \\
\pm 4.4\end{array}$ & $\begin{array}{l}551.6 \\
\pm 31.7\end{array}$ & $\begin{array}{l}155.2 \\
\pm 48.6\end{array}$ & ND \\
\hline${\mathrm{M}-D A G^{\mathrm{d}}}$ & ND & $\begin{array}{l}89.1 \\
\pm 24.7\end{array}$ & ND & $\begin{array}{l}111.9 \\
\pm 50.9\end{array}$ & $\begin{array}{l}68.3 \\
\pm 12.8\end{array}$ & ND & ND \\
\hline FFA $^{\mathrm{e}}$ & $\begin{array}{l}37.5 \\
\pm 8.8\end{array}$ & $\begin{array}{l}121.5 \\
\pm 18.1\end{array}$ & $\begin{array}{l}49.1 \\
\pm 15.6\end{array}$ & $\begin{array}{l}119.1 \\
\pm 31.1\end{array}$ & $\begin{array}{l}100.9 \\
\pm 14.0\end{array}$ & $\begin{array}{l}81.9 \\
\pm 24.7\end{array}$ & $\begin{array}{l}10.5 \\
\pm 4.3\end{array}$ \\
\hline$P L^{f}$ & ND & $\begin{array}{l}370.0 \\
\pm 53.2\end{array}$ & $\begin{array}{l}58.7 \\
\pm 30.9\end{array}$ & $\begin{array}{l}269.4 \\
\pm 48.3\end{array}$ & $\begin{array}{l}440.0 \\
\pm 75.1\end{array}$ & $\begin{array}{l}737.1 \\
\pm 129.7\end{array}$ & $\begin{array}{l}120.3 \\
\pm 35.2\end{array}$ \\
\hline
\end{tabular}

avalues reflect an average of five analyses with \pm .s.e.m. N.D $=$ not detected.

bSE, sterol esters

'TAG, triacylglycerols

dM-DAGs, mono and diacylglycerols

eFFA, free fatty acid

${ }^{f} \mathrm{~L}$, phospholipids
Although Drosophila is a sterol auxotroph, that is, it cannot synthesize these molecules (Carvalho et al. 2010), we found FAs in sterol ester fractions of rhabdomere membranes, suggesting the existence of sterol ester lipids in them. This finding concurs with the observed loss of light-dependent redistribution of the INAD (inactivation no after potential D) scaffold protein to detergent-resistant membrane fractions in response to a reduction of dietary ergosterol (Sanxaridis et al. 2007). Identification of sterol components and their nature is an interesting challenge; however, the difficulty in obtaining sufficient starting material not contaminated with other tissues has precluded a full characterization of distribution of these lipids in the rhabdomere.

Finally, while vertebrate retinas (and by extension, photoreceptors) present extremely low levels of neutral lipids (Bretillon et al. 2008; Fliesler and Anderson, 1983), our analysis of Drosophila rhabdomere membranes shows that these lipids may be particularly abundant and, interestingly, display chemical features that would make them more fluid than vertebrate neutral lipids.

By providing a characterization of the lipid composition of the membrane where the proteins involved in Drosophila phototransduction are embedded, this work fills a major gap that existed in the field, without which a thorough understanding of this physiological mechanism could not be attained.

\section{ACKNOWLEDGMENTS}

The work presented here was funded by a FONDECYT grant 1100730 (RD), MIDEPLAN ICM-P05-001 (JB), ENL11/08 (AR) and a CONICYT Master's scholarship ( $\mathrm{N}^{\mathrm{o}} 22110957$ to $\mathrm{YM}$ ).

\section{REFERENCES}

AGREN JJ, JULKUNEN A, PENTTILA I. (1992). Rapid separation of serum lipids for fatty acid analysis by a single aminopropyl column. J Lipid Res 33(12):1871-1876.

ALBERT AD, BOESZE-BATTAGLIA K. (2005). The role of cholesterol in rod outer segment membranes. Prog Lipid Res 44(2-3):99-124.

ARIKAWA K, HICKS JL, WILLIAMS DS. (1990). Identification of actin filaments in the rhabdomeral microvilli of Drosophila photoreceptors. J Cell Biol 110(6):1993-1998.

BOESZE-BATTAGLIA K, SCHIMMEL R. (1997). Cell membrane lipid composition and distribution: implications for cell function and lessons learned from photoreceptors and platelets. J Exp Biol 200 (23):2927-2936.

BRETILLON L, THURET G, GREGOIRE S, ACAR N, JOFFRE C, BRON AM, GAIN P, CREUZOT-GARCHER CP. (2008). Lipid and fatty acid profile of the retina, retinal pigment epithelium/choroid, and the lacrimal gland, and associations with adipose tissue fatty acids in human subjects. Exp Eye Res 87(6):521-528.

BROWN DA, LONDON E. (1998). Functions of lipid rafts in biological membranes. Annu Rev Cell Dev Biol 14:111-136.

CARVALHO M, SAMPAIO JL, PALM W, BRANKATSCHK M, EATON S, SHEVCHENKO A. (2012). Effects of diet and development on the Drosophila lipidome. Mol Syst Biol 8:600.

CARVALHO M, SCHWUDKE D, SAMPAIO JL, PALM W, RIEZMAN I, DEY G, GUPTA GD, MAYOR S, RIEZMAN H, SHEVCHENKO A, KURZCHALIA TV, EATON S. (2010). Survival strategies of a sterol auxotroph. Development 137(21):3675-3685.

CHYB S, RAGHU P, HARDIE RC. (1999). Polyunsaturated fatty acids activate the Drosophila light-sensitive channels TRP and TRPL. Nature 397(6716):255-259.

CLAPHAM DE. (2003). TRP channels as cellular sensors. Nature 426(6966):517-524.

DELGADO R, BACIGALUPO J. (2009). Unitary recordings of TRP and TRPL channels from isolated Drosophila retinal photoreceptor rhabdomeres: activation by light and lipids. J Neurophysiol 101(5):2372-2379. 
ESTACION M, SINKINS WG, SCHILLING WP. (2001). Regulation of Drosophila transient receptor potential-like (TrpL) channels by phospholipase C-dependent mechanisms. J Physiol 530(Pt 1):1-19.

FLIESLER SJ, ANDERSON RE. 1983. Chemistry and metabolism of lipids in the vertebrate retina. Prog Lipid Res 22(2):79-131.

FOLCH J, LEES M, SLOANE STANLEY GH. (1957). A simple method for the isolation and purification of total lipids from animal tisues. J Biol Chem 226(1):497-509.

FUJITA SC, INOUE H, YOSHIOKA T, HOTTA Y. (1987) Quantitative tissue isolation from Drosophila freeze-dried in acetone. Biochem J. 243(1):97104.

GARCIA-MURILLAS I, PETTITT T, MACDONALD E, OKKENHAUG H, GEORGIEV P, TRIVEDI D, HASSAN B, WAKELAM M, RAGHU P. (2006) lazaro encodes a lipid phosphate phosphohydrolase that regulates phosphatidylinositol turnover during Drosophila phototransduction. Neuron 49(4):533-46

HAMMAD LA, COOPER BS, FISHER NP, MONTOOTH KL, KARTY JA. (2011). Profiling and quantification of Drosophila melanogaster lipids using liquid chromatography/mass spectrometry. Rapid Common Mass Spectrum 25(19):2959-2968.

HARDIE RC, MINKE B. (1992). The trp gene is essential for a light-activated Ca2+ channel in Drosophila photoreceptors. Neuron 8(4):643-651.

HARDIE RC, POSTMA M. (2008). Phototransduction in Microvillar of Photoreceptors of Drosophila and Other Invertebrates. In: Masland R, editor. The Senses: A comprehensive Reference, Vol 1, Vision 1. 2008/09/13 ed: Academic Press. p 77-130.

HARTREE EF. (1972). Determination of protein: a modification of the Lowry method that gives a linear photometric response. Anal Biochem 48(2):422-427.

LEUNG HT, TSENG-CRANK J, KIM E, MAHAPATRA C, SHINO S, ZHOU Y, AN L, DOERGE RW, PAK WL. (2008). DAG lipase activity is necessary for TRP channel regulation in Drosophila photoreceptors. Neuron 58(6):884-96.

LEV S, KATZ B, TZARFATY V, MINKE B. (2012). Signal-dependent hydrolysis of phosphatidylinositol 4,5-bisphosphate without activation of phospholipase C: implications on gating of Drosophila TRPL (transient receptor potential-like) channel. J Biol Chem 287(2):1436-1447.

LEWIS EB. (1960). A new standard food medium. Drosphila Information Service. p 117-118.

LI-BEISSON Y, SHORROSH B, BEISSON F, ANDERSSON MX, ARONDEL V, BATES PD, BAUD S, BIRD D, DEBONO A, DURRETT TP, FRANKE RB, GRAHAM IA, KATAYAMA K, KELLY AA, LARSON T, MARKHAM JE, MIQUEL M, MOLINA I, NISHIDA I, ROWLAND O, SAMUELS L,
SCHMID KM, WADA H, WELTI R, XU C, ZALLOT R, OHLROGGE J. (2013). Acyl-lipid metabolism. Arabidopsis Book 11:e0161.

LITMAN BJ, MITCHELL DC. (1996). A role for phospholipid polyunsaturation in modulating membrane protein function. Lipids 31 Suppl:S193-197.

MCMAHON A, KEDZIERSKI W. (2010). Polyunsaturated very-long-chain C28-C36 fatty acids and retinal physiology. Br J Ophthalmol 94(9):11271132.

MONTELL C, RUBIN GM. (1989) Molecular characterization of the Drosophila trp locus: a putative integral membrane protein required for phototransduction. Neuron. 2(4):1313-23.

MUNRO S. 1995. A comparison of the transmembrane domains of Golgi and plasma membrane proteins. Biochem Soc Trans 23(3):527-530.

RAGHU P, COESSENS E, MANIFAVA M, GEORGIEV P, PETTITT T, WOOD E, GARCIA-MURILLAS I, OKKENHAUG H, TRIVEDI D, ZHANG Q, RAZZAQ A, ZAID O, WAKELAM M, O'KANE CJ, KTISTAKIS N. (2009) Rhabdomere biogenesis in Drosophila photoreceptors is acutely sensitive to phosphatidic acid levels. J Cell Biol. 185(1):129-45.

RAGHU P, YADAV S, MALLAMPATI NB. (2012). Lipid signaling in Drosophila photoreceptors. Biochim Biophys Acta 1821(8):1154-1165.

SANCHEZ-MARTIN MJ, RAMON E, TORRENT-BURGUES J, GARRIGA P. (2013). Improved conformational stability of the visual G protein-coupled receptor rhodopsin by specific interaction with docosahexaenoic acid phospholipid. Chembiochem 14(5):639-644.

SANXARIDIS PD, CRONIN MA, RAWAT SS, WARO G, ACHARYA U, TSUNODA S. (2007). Light-induced recruitment of INAD-signaling complexes to detergent-resistant lipid rafts in Drosophila photoreceptors. Mol Cell Neurosci 36(1):36-46.

SHEN LR, LAI CQ, FENG X, PARNELL LD, WAN JB, WANG JD, LI D, ORDOVAS JM,

KANG JX. (2010). Drosophila lacks C20 and C22 PUFAs. J Lipid Res 51(10):2985-2992.

WANG T, MONTELL C. (2007) Phototransduction and retinal degeneration in Drosophila. Pflugers Arch. Aug;454 (5) :821-47

TORTORIELLO G, RHODES BP, TAKACS SM, STUART JM, BASNET A, RABOUNE S, WIDLANSKI TS, DOHERTY P, HARKANY T, BRADSHAW HB. (2013) Targeted Lipidomics in Drosophila melanogaster Identifies Novel 2-Monoacylglycerols and N-acyl Amides. PLoS One 8(7):e67865.

YOSHIOKA T, INOUE H, HOTTA Y. (1985). Absence of phosphatidylinositol phosphodiesterase in the head of a Drosophila visual mutant, norpA (no receptor potential A). J Biochem 97(4):1251-1254. 